\title{
TNF biosynthesis in gut associated immunopathologies
}

Kontoyiannis D, Pasparakis M, Pizarro TT, et al. Impaired on/off regulation of TNF biosynthesis in mice lacking TNF AU-rich elements: implications for joint and gut-associated immunopathologies. Immunity 1999;10:387-98.

\section{Abstract}

We addressed the impact of deleting TNF AU-rich elements (ARE) from the mouse genome on the regulation of TNF biosynthesis and the physiology of the host. Absence of the ARE affected mechanisms responsible for TNF mRNA destabilization and translational repression in hemopoietic and stromal cells. In stimulated conditions, TNF ARE were required both for the alleviation and reinforcement of message destabilization and translational silencing. Moreover, the mutant mRNA was no longer responsive to translational modulation by the p38 and JNK kinases, demonstrating that TNF ARE are targets for these signals. Development of two specific pathologies in mutant mice, i.e., chronic inflammatory arthritis and Crohn'slike inflammatory bowel disease, suggests that defective function of ARE may be etiopathogenic for the development of analogous human pathologies.

\section{Comment}

There are a number of reasons why this paper is of interest to Gut readers. Firstly it provides new insights into the regulation of the proinflammatory cytokine tumour necrosis factor (TNF); secondly it shows that dysregulation of the TNF gene in mice leads to chronic inflammation of the joints and intestine; and thirdly there is evidence of differential roles for TNF receptors and the acquired immune response in joint and gut pathology. In addition, a useful mouse model of Crohn's disease is described.

Stability and translation of mRNA is driven by specific elements in mRNA sequences. One major class consists of adenosine-uracil multimers called AU-rich elements (ARE) which map to the 3' untranslated region (UTR) of many cytokine, oncoprotein and transcription factor transcripts. Alterations in the pattern of TNF production are associated with some diseases and the importance of TNF in rheumatoid arthritis and Crohn's disease has been demonstrated in clinical trials of TNF antagonists. The data in Kontoyiannis et al's paper suggest that defective function of its ARE could contribute to the role of TNF in disease.

A 69 base pair deletion encompassing the TNF ARE $\left(\mathrm{TNF}^{\triangle \mathrm{ARE}}\right.$ ) was targeted in mouse embryonic stem cells and mice homozygous for this construct generated. These mice were also crossed with $\mathrm{TNF}^{-/-}$mice to give monoallelic $\mathrm{TNF}^{\triangle \mathrm{ARE}}$ without the influence of normal TNF production. Appreciable levels of TNF were found in blood of mice with the mutant construct but not in controls. Macrophages from the $T \mathrm{TNF}^{\triangle \mathrm{ARE} / \mathrm{ARE}}$ mice spontaneously secreted TNF and after stimulation with lipopolysaccharide, the mRNA half life was fourfold higher than in controls. $T$ cells from $\mathrm{TNF}^{\triangle \mathrm{ARE}}$ mice also showed higher and sustained TNF- $\alpha$ release and the absence of the ARE rendered nonhaemopoietic tissues permissive for TNF production.

The weight of the TNF ${ }^{\triangle A R E / A A R E}$ mice never exceeded $7 \mathrm{~g}$ and by 5-12 weeks of age they succumbed to chronic polyarthritis and inflammatory bowel disease. The gastrointestinal tract showed a Crohn's disease-like phenotype, localised to the terminal ileum and occasionally the proximal colon. First there were mucosal abnormalities, with intestinal villus blunting and broadening. By 4 weeks of age in homozygous ( 8 weeks in hemizygous) mice there was severe intestinal inflammation deep into the muscular layers of the bowel wall with non-caseating granulomas. To investigate further the mechanisms of this immunopathology, the Kollias team crossed the $\mathrm{TNF}^{\triangle \mathrm{ARE}}$ mice with those lacking $\mathrm{TNF}$ receptors. $\mathrm{TNF}^{\triangle \mathrm{ARE} / \triangle \mathrm{ARE}} / \mathrm{TNF}-\mathrm{RI}^{-/-}$mice developed normally with no signs of illness. This is no great surprise as most of the immune pathology involving TNF is thought to be mediated by TNF-RI, which is widely expressed on most cells. TNF-RII is restricted to cells of the haemopoietic system and the phenotype of the $\mathrm{TNF}^{\triangle \mathrm{ARE} / \mathrm{ARE}} / \mathrm{TNF}-\mathrm{RII}^{-/}$mice was more intriguing. They had an even more aggressive and destructive arthritis but their gut pathology was greatly reduced. The $T N^{\triangle A R E / \triangle A R E}$ mice were also crossed with RAG1 deficient mice which lack $\mathrm{T}$ and $\mathrm{B}$ cells. Once again, the arthritic phenotype was maintained but the gut pathology abrogated.

In this model, the adaptive immune response and both TNF receptors were necessary for Crohn's disease-like pathology in the presence of deregulated TNF. By contrast, the rheumatoid arthritis phenotype may have been driven by TNF-RI expressing synoviocytes that were spontaneously producing TNF.

As yet there are no known polymorphisms of the 3' UTR of the human TNF gene. However a number of external factors, including viral proteins, can modulate the TNF ARE and hence may initiate some of the immunopathologies associated with this cytokine.

F BALKWILL Head, Biological Therapy Laboratory,
Imperial Cancer Research Fund,
Lincoln's Inn Fields,
London WC2A 3PX, UK 